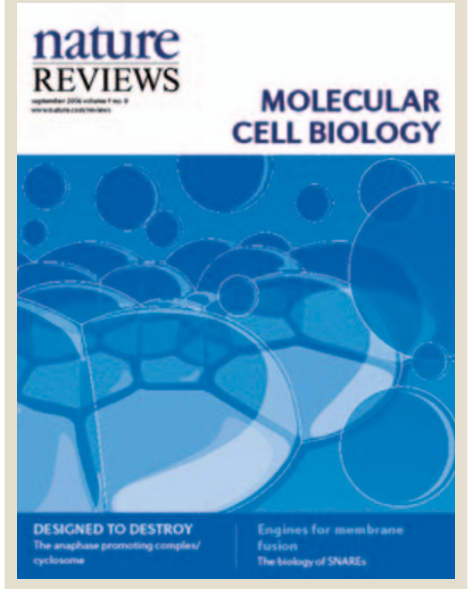

- COVER: 'Fusing structures' by Vicky Askew, inspired by the Review on $\mathrm{p} 631$

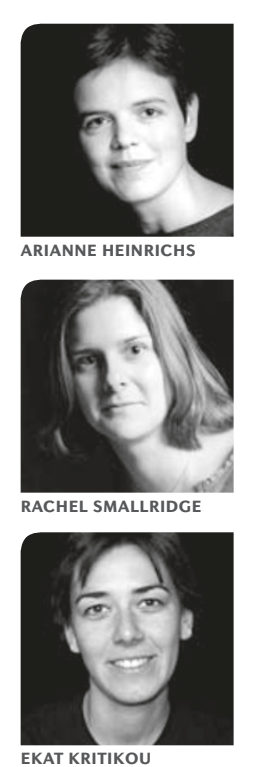

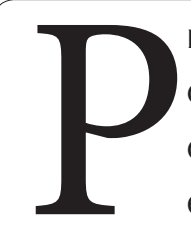
roteins rarely function alone in cells. Instead, they are usually components of larger 'molecular machines', and such dynamic complexes of proteins control many biological processes. For example, the anaphase promoting complex/cyclosome (APC/C), which contains more than a dozen subunits, is the most complex molecular machine that is known to catalyse ubiquitylation reactions. As JanMichael Peters discusses on page 644 , APC/C is a ubiquitin ligase that has important functions in the eukaryotic cell cycle. Recent work has revealed a multitude of mechanisms that control APC/C activity and has provided insights into how this intricate ubiquitin ligase recognizes its substrates.

On page 631, Reinhard Jahn and Richard H. Scheller review SNAREs (soluble $\mathrm{N}$-ethylmaleimide-sensitive factor attachment protein receptors) - robust 'nanomachines' that are key components of protein complexes that drive membrane fusion. Although still controversial, a model in which SNAREs directly function as fusion catalysts has gained wide acceptance. Furthermore, although they are small with a simple domain structure, SNAREs carry out a conformationally controlled reaction cycle of high complexity that is still not completely understood, despite recent progress.

Moving from molecular machines to the bigger picture, in an Innovation article on page 690, Rainer Pepperkok and Jan Ellenberg describe how fluorescence microscopy will be a powerful tool for systems biology because it can allow the high-throughput study of biological processes in intact living cells. In this systems era, we need to define gene function on a genome-wide scale for model organisms and humans. So, to facilitate its application to functional genomics experiments, fluorescence microscopy is making the transition to a quantitative and high-throughput technology.

\section{EDITORIAL OFFICES}

LONDON NatureReviews@nature.com

The Macmillan Building, 4 Crinan Street,

London N1 9XW, UK

Tel: +44 (0)20 78433620

Fax: $+44(0) 2078433629$

CHIEF EDITOR: Arianne Heinrichs

SENIOR EDITOR: Rachel Smallridge

ASSOCIATE EDITOR: Ekat Kritikou

ASSISTANT EDITOR: Shannon Amoils

COPY EDITOR: Asher Mullard

SENIOR ART EDITOR (NRMCB): Vicky Askew

EDITORIAL SUPPORT MANAGER:

Meg Fitzpatrick

ART CONTROLLER: Susanne Harris

SENIOR ART EDITOR/CARTOONIST:

Neil Smith

MANAGING PRODUCTION EDITOR:

Judith Shadwell

DEPUTY PRODUCTION EDITOR:

Simon Fenwick

PRODUCTION CONTROLLER: Natalie Smith
EDITORIAL ASSISTANTS: Mary Aboukhalil, Elliann Fairbairn WEB PRODUCTION EDITORS: Deborah Anthony, Angela Bird, June Lim, Amy Wagner, Nadia Younus DEPUTY WEB PRODUCTION MANAGER: DEPUTY WEB PRODU
Alexander Thurrell MARKETING MANAGER: Kellie Lane

\section{MANAGEMENT OFFICES}

LONDON nature@nature.com

The Macmillan Building, 4 Crinan Street.

London N1 9XW, UK

Tel: +44 (0)20 7833 4000;

Fax: +44 (0)20 $78434596 / 7$

OFFICE MANAGER: Sheryl Ocampo

PUBLISHER: Hugh Blackbourn

MANAGING DIRECTOR: Annette Thomas

EDITOR-IN-CHIEF, NATURE PUBLICATIONS:

EDITOR-IN-CHIEF,
Philip Campbell

ASSOCIATE DIRECTORS:

Jenny Henderson, Tony Rudland
EDITORIAL PRODUCTION DIRECTOR: James McQuat

PRODUCTION MANAGER: Yvonne Strong WEB PRODUCTION MANAGER, UK:

Amanda Ward

DIRECTOR, WEB PUBLISHING:

Timo Hannay

HEAD OF WEB PRODUCTION:

Jeremy Macdonald

NEW YORK nature@natureny.com

Nature Publishing Group, 75 Varick Street

9th floor, New York, NY 10013-1917, USA

Tel: +1 212726 9200;

Fax: +12126969006

CHIEF TECHNOLOGY OFFICER:

Howard Ratner

DIRECTOR OF NEW TECHNOLOGY:

Greg Suprock

HEAD OF WEB SERVICES: Anthony Barrera

NATUREJOBS PUBLISHER: Ben Crowe

HEAD OF NATURE RESEARCH \& REVIEWS

MARKETING: Sara Girard
TOKYO nature@natureasia.com

Chiyoda Building 5F, 2-37-1 Ichigayatamachi,

Shinjuku-ku, Tokyo 162-0843, Japan

Tel: +81 33267 8751;

Fax: +81332678746

ASIA-PACIFIC PUBLISHER:

Antoine E Bocquet

MANAGER: Koichi Nakamura

ASIA-PACIFIC SALES DIRECTOR:

Kate Yoneyama

SENIOR MARKETING MANAGER:

Peter Yoshihara

MARKETING/PRODUCTION MANAGER:

Takesh Murakami

INDIA 5A/12 Ansari Road, Daryganj,

New Delhi 110 002, India

Tel/Fax: +91112324 4186

SALES AND MARKETING MANAGER, INDIA:

Harpal Singh Gill

Copyright $\odot 2006$ Nature Publishing Group

Printed in Wales by Cambrian Printers

on acid-free paper 